L.A. BULAVIN, G.M. VERBINS'KA, D.A. GAVRYUSHENKO, V.M. SYSOEV, O.S. SOLOV'YOV, K.V. CHEREVKO

Taras Shevchenko National University of Kyiv, Faculty of Physics

(64, Volodymyrs'ka Str., Kyiv 01601, Ukraine; e-mail: vovkulaka3d@gmail.com)

PACS 05.70.-a; 05.70.Ln; $66.10 . \mathrm{cg}$

\section{ENTROPY PRODUCTION IN THE DIFFUSION-DRIVEN REGIME OF DROPLET EVAPORATION}

\begin{abstract}
A consistent model describing the evaporation of a spherical droplet has been developed. Expressions for the total flux and production of entropy have been obtained in the case of ideal solution with regard for the concentration dependence of the diffusion coefficient. The results obtained allow the stabilizing effect for the entropy production and flux to be revealed even in the case where only the entropy contributions to thermodynamic potentials at the mixing are taken into account.

Ke ywords: entropy production, droplet evaporation
\end{abstract}

\section{Introduction}

The development of a consistent thermodynamic theory that could describe the process of diffusioncontrolled evaporation of a spherical droplet proceeding from the basic ideas of the thermodynamics of nonequilibrium processes - in particular, with regard for the concentration dependence of the diffusion coefficient of an evaporated material in the gaseous environment - is a challenging problem from both the experimental and theoretical viewpoints. Moreover, due to a rapid progress in nanotechnologies attained for the last years, it is of interest to study the evaporation of liquids in carbon nanotubes. Owing to the surface tension, the free surface of such liquids in nanotubes has a curved shape, so that this surface can be considered in the first approximation as a hemisphere, with the liquid being evaporated from it. The hemisphere radius is governed not only by the nanotube diameter, but also by the surface tension coefficient of a liquid in the nanotube. In turn, for characteristic sizes of nanotubes, this coefficient substantially depends on the nanotube diameter. Therefore, while considering the evaporation processes under those conditions, it is pertinent to consider a variation of the droplet radius induced by surface tension forces. When calculating the thermodynamic parameters of the system, it is also important to take a nonuniformity of the temperature and the pressure into consideration.

(C) L.A. BULAVIN, G.M. VERBINS'KA,

D.A. GAVRYUSHENKO, V.M. SYSOEV,

O.S. SOLOV'YOV, K.V. CHEREVKO, 2013

\section{Entropy Production in the Diffusion-Driven Regime of Droplet Evaporation}

Consider the following model. A spherical droplet of radius $r_{1}$ is surrounded by the gaseous medium, a background gas that is insoluble in the droplet substance (in other words, the background gas flux across the droplet surface equals zero). We suppose that, in the course of evaporation, the droplet remains to be a ball with fixed radius, and there are no external fields and chemical reactions in the system. In some time, owing to the evaporation process, the gas around the droplet will be a mixture of the background gas (we denote its concentration by $x_{2}$ ) and the gas created by the droplet substance (the concentration of this gas is denoted by $x_{1}$ ). The evaporation process is considered to be stationary. Let the droplet be maintained at a constant temperature $T_{r_{1}}$ by a heat source located inside, and let there be no external factors that would create temperature gradients in the system. We consider the case where the evaporation process does not affect the pressure in the system; in other words, we suppose that the gradient of the total substance density in the system equals zero. The formulated problem evidently has a spherical symmetry. Therefore, we will consider it in a spherical coordinate system with the origin at the droplet center. Then, the system of equations that describe the process of diffusion-controlled evaporation from the droplet can be represented as Fick's law, in which the concentration gradient is taken 
into account:

$$
\left\{\begin{array}{l}
J=-\frac{L_{1}}{T}\left[\frac{\partial \mu_{1}}{\partial x_{1}} \frac{\partial x_{1}}{\partial r}+\frac{\partial \mu_{2}}{\partial x_{2}} \frac{\partial x_{1}}{\partial r}+\left(\frac{\partial \mu_{1}}{\partial T}-\frac{\mu_{1}}{T}\right) \frac{d T}{d r}\right] \\
0=-\frac{L_{2}}{T}\left[\frac{\partial \mu_{2}}{\partial x_{1}} \frac{\partial x_{1}}{\partial r}+\frac{\partial \mu_{2}}{\partial x_{2}} \frac{\partial x_{1}}{\partial r}+\left(\frac{\partial \mu_{2}}{\partial T}-\frac{\mu_{2}}{T}\right) \frac{d T}{d r}\right] \\
0=\frac{d x_{1}}{d r}+\frac{d x_{2}}{d r}
\end{array}\right.
$$

Here, $J_{1}$ is the evaporated substance flux; $L_{1}$ and $L_{2}$ are the kinetic coefficients [1] of the evaporated substance and the background gas, respectively; and $\mu_{1}$ and $\mu_{2}$ are the corresponding chemical potentials.

Let the concentration of evaporated substance be maintained at a stationary level equal to $x_{r_{1}}$ near the droplet surface and to $x_{r_{2}}$ at a certain distance $r_{2}$ from the droplet center, which is large enough in comparison with $x_{r_{1}}$. Then, the following boundary conditions can be written down for the evaporated substance concentration:

$x_{1}\left(r_{1}\right)=x_{r_{1}}, \quad x_{1}\left(r_{2}\right)=x_{r_{2}}$.

For the temperature, the boundary conditions are formally expressed in the form

$T\left(r_{1}\right)=T_{1}, \quad T\left(r_{2}\right)=T_{2}$,

where $T_{2}$ is a temperature, which is not a parameter of the problem, but a quantity to be determined from the solution of the system of equations.

The system of equations (1) can be presented as two differential equations. One of them relates the temperature gradient in the system to the gradient of the evaporated substance concentration [1],

$$
\frac{d T}{d r}=-2 \frac{\frac{\partial \mu_{2}}{\partial T}}{\frac{\partial \mu_{2}}{\partial T}-\frac{\mu_{2}}{T}} \frac{d x_{1}}{d r},
$$

and the other relates the gradient of the evaporated substance concentration to the substance flux,

$J_{1}=-2 \frac{L_{1}}{T}\left[\frac{\partial \mu_{1}}{\partial x_{1}}-\frac{\partial \mu_{2}}{\partial x_{1}} \frac{\frac{\partial \mu_{1}}{\partial T}-\frac{\mu_{1}}{T}}{\frac{\partial \mu_{2}}{\partial T}-\frac{\mu_{2}}{T}}\right] \frac{d x_{1}}{d r}$.

Using the expression $\mu_{i}=\mu_{i 0}+k_{\mathrm{B}} T \ln \left(\gamma_{i} x_{i}\right)$, where $\gamma\left(T, P, x_{1}, x_{2}, \ldots, x_{n}\right)$ is the so-called activity coefficient, for the chemical potential of the solution,
Eqs. (4) and (5) take the final form

$$
\begin{aligned}
& \frac{d T}{d r}=-2 k_{\mathrm{B}} T \frac{\frac{1}{1-x_{1}}-\frac{\partial}{\partial x_{1}} \ln \gamma_{2}}{-s_{20}-\frac{\partial \mu_{20}}{\partial T}+k_{\mathrm{B}} T \frac{\partial}{\partial T} \ln \gamma_{2}} \frac{d x_{1}}{d r}, \\
& J_{1}=-2 k_{\mathrm{B}} L_{1}\left[\frac{1}{x_{1}}+\frac{\partial}{\partial x_{1}}\right] \frac{d x_{1}}{d r}- \\
& -2 k_{\mathrm{B}} L_{1}\left[\frac{1}{1-x_{1}}+\frac{\partial}{\partial x_{1}}\right] \times \\
& \times\left[\frac{-s_{10}-\frac{\partial \mu_{10}}{\partial T}+k_{\mathrm{B}} T \frac{\partial}{\partial T} \ln \gamma_{1}}{-s_{20}-\frac{\partial \mu_{20}}{\partial T}+k_{\mathrm{B}} T \frac{\partial}{\partial T} \ln \gamma_{2}}\right] \frac{d x_{1}}{d r} .
\end{aligned}
$$

By squaring the expression for the flux, we obtain the following formula for the entropy production:

$$
\begin{aligned}
& \sigma=L_{1}\left(2 k_{\mathrm{B}}\right)^{2}\left(\frac{d x_{1}}{d r}\right)^{2}\left[\frac{1}{x_{1}}+\frac{\partial}{\partial x_{1}} \ln \gamma_{1}+\right. \\
& \left.+\left(\frac{1}{1-x_{1}}-\frac{\partial}{\partial x_{1}} \ln \gamma_{2}\right) \frac{-s_{10}-\frac{\partial \mu_{10}}{\partial T}+k_{\mathrm{B}} T \frac{\partial}{\partial T} \ln \gamma_{1}}{-s_{20}-\frac{\partial \mu_{20}}{\partial T}+k_{\mathrm{B}} T \frac{\partial}{\partial T} \ln \gamma_{2}}\right]^{2},
\end{aligned}
$$

where $s_{i 0}$ is the specific entropy (per particle) in the pure $i$-th component.

Equations (6), (7), and (8) together with conditions (2) and (3) allow the spatial profiles of the evaporated substance concentration and the temperature to be plotted, and the substance flux $J_{1}$ and the entropy production $\sigma$ to be determined. For this purpose, the equation for the gas mixture around the droplet has evidently to be specified. Note that the temperature gradient in the system is not supported from outside. Nevertheless, despite that fact and owing to the droplet evaporation, the gradient of concentration $\frac{d x_{1}}{d r}$ and, as a result, the gradient of temperature emerge across the system. The latter can be determined with the help of expression (4). The existence of such a gradient is confirmed by modern experimental data on the droplet evaporation [2].

\section{Entropy Production}

\section{in the Diffusion-Controlled Regime of Droplet} Evaporation. The Case of Ideal Gas

Consider the case where the gas mixture consisting of the background and evaporated gases is ideal. The contribution of entropy factors to thermodynamic potentials at the mixing dominates over those made by energy ones, so that the latter can be neglected.

ISSN 2071-0194. Ukr. J. Phys. 2013. Vol. 58, No. 2 
In view of the formula $\mu_{i}=\mu_{i 0}++k_{\mathrm{B}} T \ln \left(1-x_{i}\right)$ for the chemical potential of the ideal gas (i.e. the activity coefficient $\gamma=1$ ), Eq. (7) can be presented in the form [3]

$J_{1}=-2 L_{1} k_{\mathrm{B}}\left[\frac{1}{x_{1}}+\frac{1}{1-x_{1}}\right] \frac{x_{1}}{d r}$.

Note that, while determining the flux in the case of a mixture of ideal gases, there is no need to calculate the spatial distribution of the temperature over the medium that surrounds the droplet. The solution of Eq. (9) with regard for formal boundary conditions (2) and (3) looks like

$J_{1}=-2 L_{1} k_{\mathrm{B}} \frac{r_{1} r_{2}}{r_{2}-r_{1}} \frac{1}{r^{2}}\left[\ln \frac{x_{r_{2}}}{x_{r_{1}}}-\ln \frac{1-x_{r_{2}}}{1-x_{r_{=1}}}\right]$.

It is important to emphasize that, according to this expression obtained in the approximation of the mixture of ideal gases, the evaporated substance flux does not depend on the ratios between such gas parameters as their molar masses or partial volumes [4].

In Fig. 1, the dependences of the normalized flux $-\frac{J_{1}}{2 k_{\mathrm{B}} L_{1}} \frac{r_{2}-r_{1}}{r_{1} r_{2}} r^{2}$ on the difference between the given concentrations $x_{r_{1}}$ and $x_{r_{2}}$ are plotted for various $x_{r_{2}}$. The results obtained testify to a considerable nonlinearity in the flux dependence on the quantity $x_{r_{1}}-x_{r_{2}}$. The analysis of the presented results shows that an almost linear dependence of the flux is observed at small $x_{r_{1}}-x_{r_{2}}$ values, but the dependence $J_{1}\left(x_{r_{1}}-x_{r_{2}}\right)$ substantially deviates from the linear one if the difference $x_{r_{1}}-x_{r_{2}}$ grows further, and the stabilizing effect takes place, i.e. the flux becomes weaker dependent on the difference between the concentrations at the system boundaries.

For the entropy production in the case concerned, we obtain the expression

$\sigma=\left(2 k_{\mathrm{B}}\right)^{2} L_{1}\left(\frac{r_{1} r_{2}}{r_{2}-r_{1}}\right)^{2} \frac{1}{r^{4}}\left[\ln \frac{x_{r_{2}}}{x_{r_{1}}}-\ln \frac{1-x_{r_{2}}}{1-x_{r_{1}}}\right]^{2}$.

In Fig. 2, the dependences of the normalized entropy production $\sigma_{n}=\frac{\sigma}{\left(2 k_{\mathrm{B}}\right)^{2} L_{1}}\left(\frac{r_{2}-r_{1}}{r_{1} r_{2}}\right)^{2} r^{4}$ on the difference between the given concentrations $x_{r_{1}}$ and $x_{r_{2}}$ are plotted for various $x_{r_{2}}$. The results point to a considerable deviation of the dependence concerned from the square law. We should emphasize that the entropy production in the approximation of a mixture of ideal gases is also independent of the ratios

ISSN 2071-0194. Ukr. J. Phys. 2013. Vol. 58, No. 2

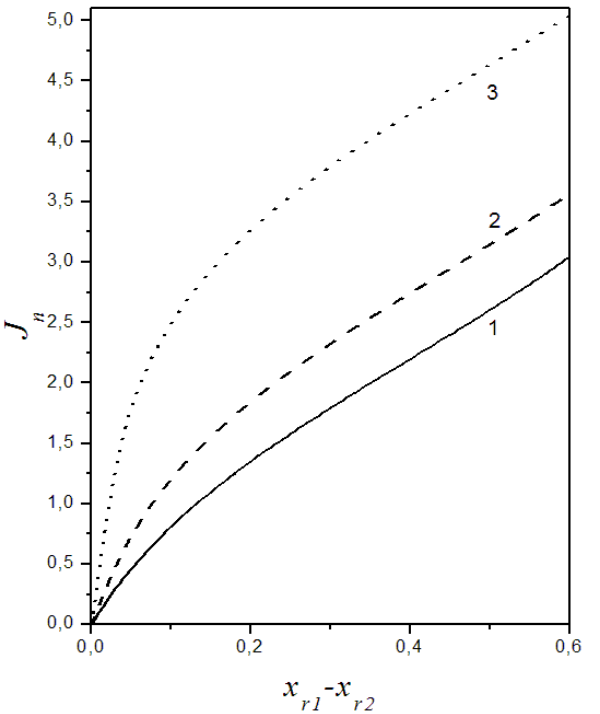

Fig. 1. Dependences of the normalized flux $J_{n}=-\frac{J_{1}}{2 k_{\mathrm{B}} L_{1}} \times$ $\times \frac{r_{2}-r_{1}}{r_{1} r_{2}} r^{2}$ on the difference $x_{r_{1}}-x_{r_{2}}$ for various $x_{r_{2}}=0.1$ (1), $0.05(\stackrel{2}{2})$, and 0.01 (3). $x_{r_{1}}=0.7$

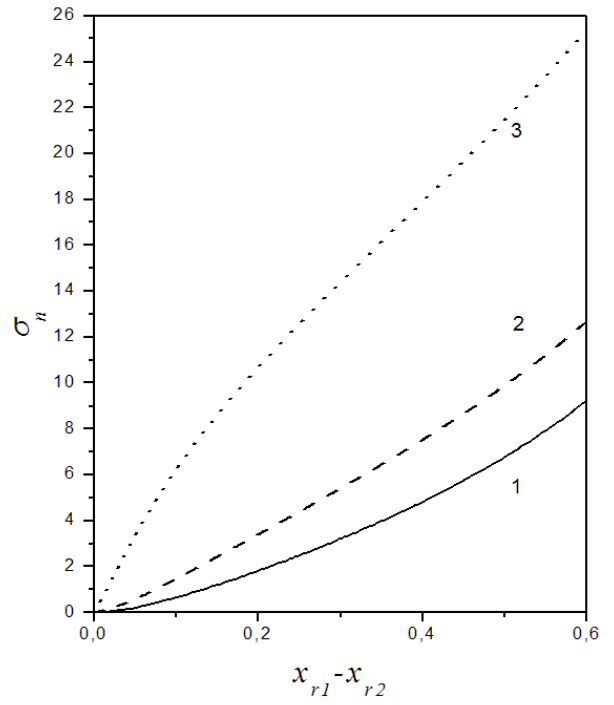

Fig. 2. Dependences of the normalized entropy production $\sigma_{n}=\frac{\sigma}{\left(2 k_{\mathrm{B}}\right)^{2} L_{1}}\left(\frac{r_{2}-r_{1}}{r_{1} r_{2}}\right)^{2} r^{4}$ on the difference $x_{r_{1}}-x_{r_{2}}$ for various $x_{r_{2}}=0.1$ (1), 0.05 (2), and 0.01 (3). $x_{r_{1}}=0.7$

between such parameters of the gases as their molar masses or partial volumes.

Hence, even considering only the entropy contributions to the thermodynamic potentials at the mixing already gives rise to considerable deviations of the calculated results from the classical ones obtained in the framework of the approximation of a constant dif- 
fusion coefficient. However, if the droplet evaporation is simulated, the consideration of the surrounding gas as a mixture of ideal gases does not allow the thermodynamic and molecular kinetic features of mixture components to be taken into account correctly.

Moreover, the magnitudes of both the diffusion flux (see expression (10)) and the entropy production (see expression (11)) depend on the droplet radius. The latter can be determined by the surface tension coefficient, which, in turn, depends on the system size in the case of nano-scaled systems.

\section{Conclusions}

Provided that the flux of a background gas is absent, the flux of the evaporated substance is compensated by the emergence of a temperature gradient in the system. In the course of droplet evaporation, the considerable effect of a flux stabilization manifests itself in the system even if only the entropy contributions to a variation of the thermodynamic potentials at the mixing are taken into account. As a result, the dependence of the evaporated substance flux on the concentration acquires a substantially nonlinear form. If only the entropy contributions to a variation of the thermodynamic potentials at the mixing are considered, the entropy production at the droplet evaporation becomes much less sensitive to a variation of the concentration gradient the diffusing substance.
1. S.R. de Groot and P. Mazur, Non-Equilibrium Thermodynamics (North-Holland, Amsterdam, 1962).

2. D.A. Gavryushenko, V.M. Sysoev, and K.V. Cherevko, in Modern Problems of Molecular Physics (Kyiv University Publ. House, Kyiv, 2006), p. 181 (in Ukrainian).

3. C.A. Ward and G. Fang, Phys. Rev. F 59, 429 (1998).

4. D.A. Gavryushenko, V.M. Sysoev, and K.V. Cherevko, in Proceedings of the 11-th Russian Conference on Thermal Material Properties (Saint-Petersburg, 2005), p. 21 (in Russian).

5. D.A. Gavryushenko, V.M. Sysoev, and K.V. Cherevko, Ukr. J. Phys. 52, 30 (2007).

Received 26.04.12.

Translated from Ukrainian by O.I. Voitenko

Л.А. Булавін, Г.М. Вербінсъка, Д.А. Гаврюшенко, B.М. Сисоєв, О.С. Соловйов, К.В. Черевко

ВИРОБНИЦТВО ЕНТРОПІЇ В ДИФУЗІЙНОМУ РЕЖИМІ ВИПАРОВУВАННЯ КРАПЛИНИ

$\mathrm{P}$ е $з$ ю м е

Побудовано несуперечливу модель для випаровування сферичної краплини. Отримано вираз для сумарного потоку і виробництва ентропії у випадку ідеального розчину, 3 урахуванням концентраційної залежності коефіцієнта дифузії. Отримані результати дозволяють виявити стабілізуючий ефект за потоком навіть при врахуванні виключно ентропійних внесків у зміну термодинамічних потенціалів під час змішування. 